\title{
Management of Appendix Cancer
}

\author{
Kaitlyn J. Kelly, MD ${ }^{1}$ \\ ${ }^{1}$ Department of Surgery, University of California San Diego, San \\ Diego, California \\ Clin Colon Rectal Surg 2015;28:247-255.
}

\begin{abstract}
Address for correspondence Kaitlyn J. Kelly, MD, Division of Surgical Oncology, Moores Cancer Center, University of California, San Diego, 3855 Health Sciences Drive, Mail code 0987, La Jolla, CA 92093-0987 (e-mail: k6kelly@ucsd.edu).
\end{abstract}

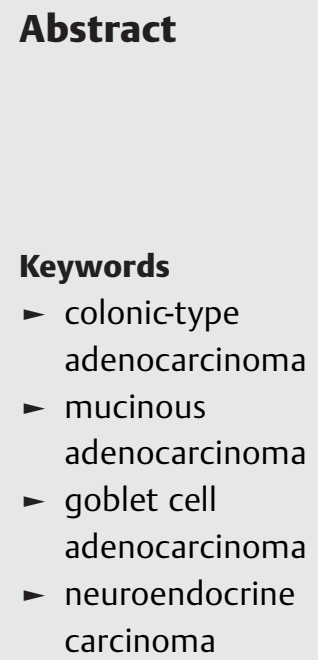

Primary cancers of the appendix are rare and are frequently diagnosed after surgery for appendicitis, presumed ovarian primary malignancy, or other indications. Primary appendix cancers are histologically diverse, and classification of these tumors has historically been confusing because of the nonstandardized nomenclature that is used. This review aimed to describe the epidemiology, presentation, workup, staging, and management of primary appendix cancers using current, recommended nomenclature. For this purpose, tumors were broadly classified as colonic-type or mucinous adenocarcinoma, goblet cell adenocarcinoma, or neuroendocrine carcinoma. Signet ring cell carcinoma was not regarded as an individual entity. The presence of signet ring cells is a histologic feature that may or may not be present in colonic-type or mucinous adenocarcinoma. The management of primary appendix cancer is complex and is dependent on the histologic subtype and extent of disease. Randomized, prospective trials do not exist for these rare tumors and management is largely guided by retrospective data expert consensus guidelines, which are summarized here.
Primary cancers of the appendix are rare, with an incidence of approximately 1.2 cases per 100,000 people per year in the United States. ${ }^{1}$ There are no established risk factors for the development of appendix cancer. Malignant appendix tumors most often present with acute appendicitis and are diagnosed incidentally at histologic assessment of the surgical specimen. Appendix cancers may also be asymptomatic and be found incidentally as an abnormal-appearing appendiceal orifice on colonoscopy, at surgery, or on cross-sectional imaging for other indications, such as presumed ovarian malignancy. When symptoms are present, the disease process is often advanced. For example, many patients present with abdominal distention and pain secondary to peritoneal dissemination of appendix cancer.

For such a small organ, the appendix gives rise to surprisingly diverse morphologic tumor types. The differential diagnosis of primary appendix cancer includes adenocarcinoma, neuroendocrine carcinoma, and mixed tumors containing both of these elements with goblet cells. There is tremendous histologic and biologic heterogeneity within these broad categories, with tumors ranging from low to high

Issue Theme Colon Cancer; Guest Editor: Garrett M. Nash, MD, MPH, FACS, FASCRS grade, with or without signet ring cells. Given this morphologic diversity, classification of appendiceal tumors has historically been confusing. For the purposes of this review, appendix cancers will be broadly classified as: colonic-type adenocarcinoma, mucinous adenocarcinoma, goblet cell adenocarcinoma (GCA), and neuroendocrine carcinoma (aka. "typical carcinoid").

\section{Colonic-Type Adenocarcinoma}

\section{Background and Epidemiology}

Overall, adenocarcinoma is thought to be the most common type of primary appendix cancer, comprising $60 \%$ of all cases. ${ }^{1}$ Nevertheless, it constitutes less than $0.5 \%$ of all gastrointestinal tract neoplasms. ${ }^{1,2}$ Primary appendix adenocarcinoma is classified as "colonic-type," which arises from preexisting adenomas similar to colorectal tumors, or "mucinous," which is biologically and histologically distinct from colonic adenocarcinoma. ${ }^{3}$ Similar to colon cancer, colonic-type adenocarcinoma of the appendix presents at a mean age of 62 to 65 years, with a slight male sex predominance. ${ }^{1,4}$ Given the
Copyright (c) 2015 by Thieme Medical Publishers, Inc., 333 Seventh Avenue, New York, NY 10001, USA.

Tel: +1(212) 584-4662.
DOI http://dx.doi.org/ 10.1055/s-0035-1564433. ISSN 1531-0043. 
rarity of this disease, there is no designated American Joint Commission on Cancer (AJCC) staging system or National Comprehensive Cancer Network (NCCN) evidence-based guideline specific for it. The workup, staging, and treatment of colonic-type adenocarcinoma arising in the appendix mirror that of colon cancer.

\section{Presentation and Workup}

Patients with colonic-type adenocarcinoma of the appendix most often present with incidentally identified lesions following appendectomy for appendicitis or other indication, and will have pathologic T-stage information immediately available. Tis tumors resected with negative margins can be managed with appendectomy alone. T1 tumors with favorable characteristics are thought of like malignant polyps. Appendectomy alone may be sufficient if these lesions are grade 1 or 2 , have no angiolymphatic invasion, and have negative resection margins. All patients should undergo complete colonoscopy to evaluate for synchronous colorectal lesions.

\section{Management}

Patients found to have unfavorable T1 tumors (high-grade, angiolymphatic invasion, and/or positive margins) should be considered for formal right hemicolectomy for adequate staging and resection. Patients with T2 or greater tumors require complete staging with contrast-enhanced computed tomography (CT) of the chest, abdomen, and pelvis if not already performed. If there is no evidence of distant metastasis, right hemicolectomy is recommended, with 12 or more lymph nodes typically considered adequate for accurate staging. In the largest population-based study of primary appendix cancer, the rate of lymph node involvement in the colonic-type adenocarcinoma subtype was 30\%. ${ }^{1}$ Patients with node-positive disease (stage III) warrant adjuvant systemic chemotherapy with 5-fluorouracil/leucovorin or capecitabine with oxaliplatin if medically fit. As for colonprimary adenocarcinoma, adjuvant chemotherapy should also be considered for stage II patients with high-risk features, especially younger patients and those with inadequate nodal staging.

The rate of distant metastasis at presentation for colonictype adenocarcinoma of the appendix is not well known given the rarity of the disease, but has been reported at 23 to $37 \%{ }^{1,4}$ The peritoneum (including ovaries) is the most common site, and the liver and lung metastases are less common. Systemic chemotherapy is the recommended treatment for asymptomatic patients who present with distant metastasis. For patients with peritoneal-only metastatic disease, complete cytoreductive surgery and intraperitoneal (IP) chemotherapy should be considered if complete tumor debulking can be achieved. Cytoreduction and IP chemotherapy are discussed in detail in the next section on mucinous adenocarcinoma in which isolated peritoneal metastasis is more common. As is true for colorectal cancer, surgical resection, including metastasectomy of limited liver or lung lesions, is reasonable for select patients with appendix adenocarcinoma.

\section{Mucinous Adenocarcinoma}

\section{Background and Epidemiology}

Mucinous adenocarcinoma of the appendix (MAA) appears to be a biologically and histologically distinct entity from colorectal cancer and from colonic-type adenocarcinoma of the appendix. The mean age at presentation is 60 years and there is no clear sex predilection. ${ }^{1,4}$ There are no known risk factors for this disease. MAA is broadly classified histologically as low or high grade, and this distinction is greatly important for determining prognosis and treatment. The 7th edition of AJCC staging system for MAA is shown in - Table 1.

\section{Presentation and Workup}

Patients with MAA most often present after rupture of the primary tumor with spread of mucin and tumor cells throughout the peritoneal cavity. The resulting clinical syndrome of abdominal distention secondary to mucinous ascites is known as pseudomyxoma peritonei (PMP) and is usually diagnosed at operation for appendicitis, peritonitis, or presumed ovarian malignancy, or on cross-sectional imaging. MAA tends to remain inside of the peritoneal cavity with extraperitoneal (including lymphatic) metastasis seen only in rare cases. For this reason, peritoneal disease may be considered a regional, rather than distant, metastatic site.

Low-grade MAA (G1) and low grade appendiceal mucinous neoplasm (LAMN) are characterized by well-differentiated, glandular, mucin-producing cells. Despite being classically described as "noninvasive" histologically, these cells still lead to PMP and are considered malignant. Unlike LAMN, low-grade MAA may exhibit gross invasion of involved organs, as evident by the gross photo of a distal gastrectomy specimen removed during cytoreduction for low-grade MAA in - Fig. 1A. This image shows densely adherent tumor deposits that could not be separated from the stomach, but that still appeared low-grade on the microscopic level. However, low-grade MAA tends to be indolent and slowly progressive. Patients may have considerable disease burden but may live for many years with minimal symptoms. -Fig. 1B demonstrates a cross-sectional image from this same patient who had been living with slowly progressive disease over the course of 5 years since her initial diagnosis with minimal symptoms. A three-tiered grading system for MAA has been proposed that accounts for adverse features such as destructive invasion, angiolymphatic invasion, high cellularity, perineural invasion, and signet ring cell component in addition to cytologic grade. ${ }^{5}$ While this system has not yet been widely adopted, it may prove to better stratify those patients with purely noninvasive disease from those with more intermediate, indolent but invasive disease like the patient described here.

High-grade MAA is characterized by invasive adenocarcinoma cells that may or may not have a signet ring cell component. High-grade MAA exhibits a much more aggressive clinical course than low-grade. Patients are more often symptomatic at presentation with complaints such as unintentional weight loss, pain, bloating, and bowel obstruction.

In both low- and high-grade MAA, assessment of extent of disease is the first step in the workup when patients present with peritoneal dissemination. The peritoneal carcinomatosis 
Table 1 AJCC staging (7th edition) of mucinous adenocarcinoma of the appendix

\begin{tabular}{|l|l|l|l|}
\hline T stage & & Stage & \\
\hline Tx & Primary tumor cannot be assessed & 0 & Tis, N0, M0 \\
\hline Tis & Carcinoma in situ & I & T1/2, N0, M0 \\
\hline T1 & Invades submucosa & IIA & T3, N0, M0 \\
\hline T2 & Invades muscularis propria & IIB & T4a, N0, M0 \\
\hline T3 & Invades through muscularis propria & IIC & T4b, N0, M0 \\
\hline T4a & Invades visceral peritoneum & IIIA & T1/2, N1, M0 \\
\hline T4b & Invades adjacent organs & IIIB & T3/4, N1, M0 \\
\hline Nodes & & IIIC & Any T, N2, M0 \\
\hline N0 & No regional lymph node involvement & IVA & Any T, N0, Mla, G1 \\
\hline N1 & Metastasis to 1-3 regional nodes & IVB & Any T, N0, Mla, G2/3 \\
\hline N2 & Metastasis to $\geq 4$ regional nodes & & Any T, N1/2, Mla, any G \\
\hline Metastasis & & IVC & Any T, any N, MIb, any G \\
\hline M0 & No distant metastasis & & \\
\hline M1a & Intraperitoneal-only metastasis & & \\
\hline M1b & Extraperitoneal distant metastasis & & \\
\hline Grade & & & \\
\hline GX & Cannot be determined & & \\
\hline G1 & Well-differentiated & & \\
\hline G2 & Moderately differentiated & & \\
\hline G3 & Poorly differentiated & & \\
\hline G4 & Undifferentiated & & \\
\hline
\end{tabular}

Abbreviation: AJCC, American Joint Commission on Cancer.

index (PCI) described by Jacquet and Sugarbaker is the most commonly used metric for assessment of disease burden (- Fig. 2). ${ }^{6} \mathrm{PCl}$ can be estimated preoperatively by contrastenhanced cross-sectional imaging with computed tomography (CT) or magnetic resonance imaging (MRI). However, small peritoneal deposits are difficult to appreciate on CT and in some cases it can be difficult to distinguish between mucin and tumor. There is some evidence suggesting that diffusionweighted MRI may be superior to $\mathrm{CT}$ in evaluating extent of peritoneal disease. $^{7}$ Nevertheless, both MRI and CT are acceptable for preoperative assessment of disease burden.

If the estimated preoperative $\mathrm{PCl}$ is greater than 20 , in the presence of high-grade histology, complete surgical resection is more challenging. Additional findings that predict the ability to perform a complete surgical resection include extensive small bowel involvement, evident by diffuse thickening and hyperenhancement of the bowel wall, small bowel obstruction, hydronephrosis or ureteral obstruction, and

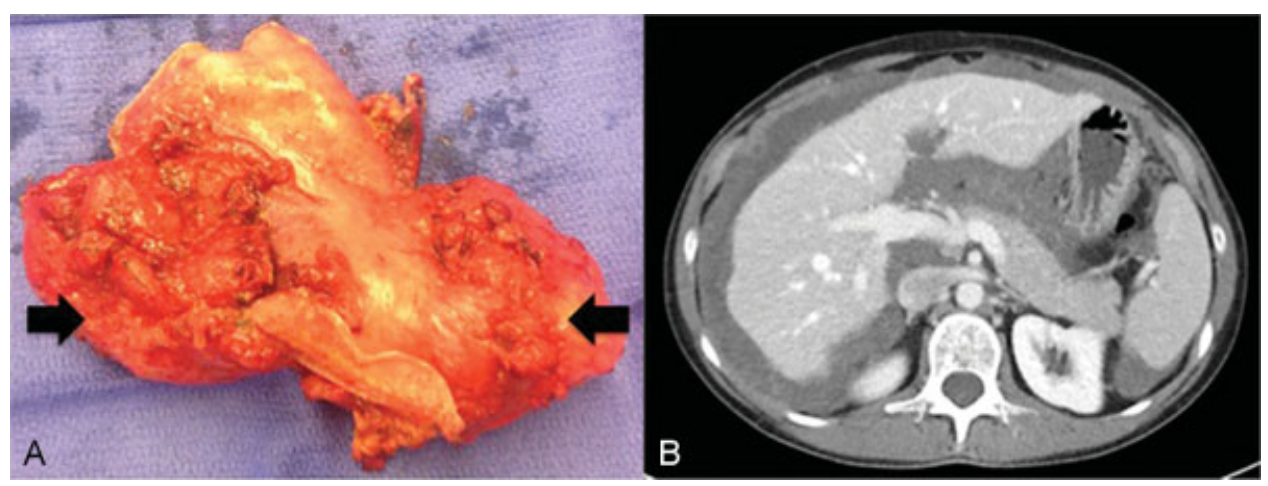

Fig. 1 (A) Gross photograph of distal gastrectomy specimen involved by low-grade MAA. Despite being low-grade, the tumor exhibited gross tissue invasion. (B) CT scan from the same patient demonstrating extensive low-grade MAA that was minimally symptomatic. 


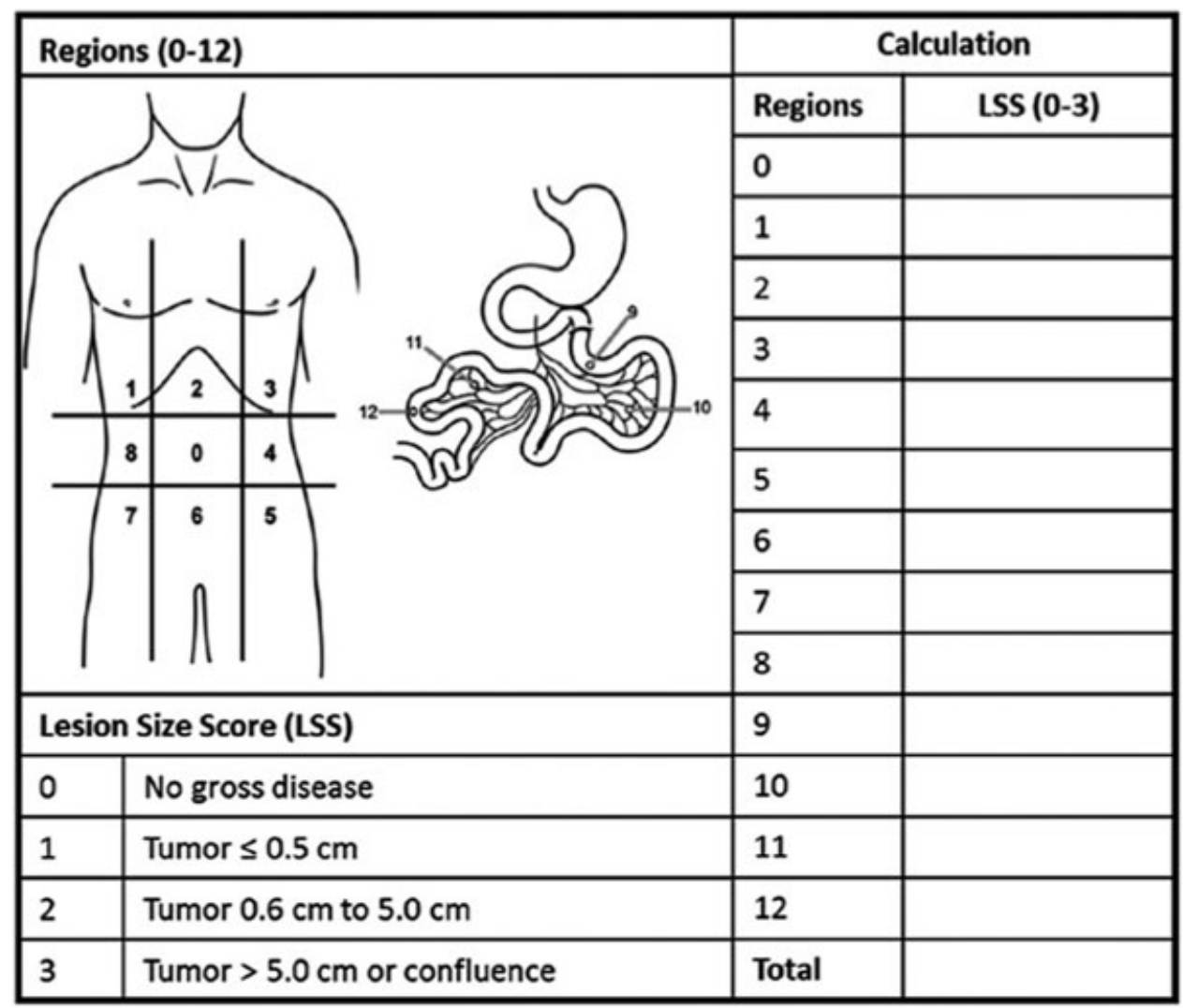

Fig. 2 Peritoneal carcinomatosis index $(\mathrm{PCl})$. $\mathrm{PCl}$ is determined by assigning a $\mathrm{LSS}$ to each of the 13 peritoneal regions and summing the scores. LSS, lesion size score. The maximum possible score is 39. (Reprinted with permission from Kelly KJ, Nash GM. Peritoneal debulking/intraperitoneal chemotherapy-Non-sarcoma. J Surg Oncol 2014;109:14-22.)

extensive upper abdominal disease with encasement or deformity of vital structures such as the porta hepatis or inferior vena cava. Last, extraperitoneal metastatic disease to liver parenchyma, retroperitoneal lymph nodes, or other sites is considered by many to be a contraindication to curativeintent surgery.

\section{Management}

Management of MAA is dependent on the histologic type and the extent of disease at presentation, and is outlined in - Fig. 3. For those fortunate patients with MAA that has not ruptured, complete surgical resection with appendectomy without spillage of mucin is recommended. - Fig. 4 illustrates a CT scan from

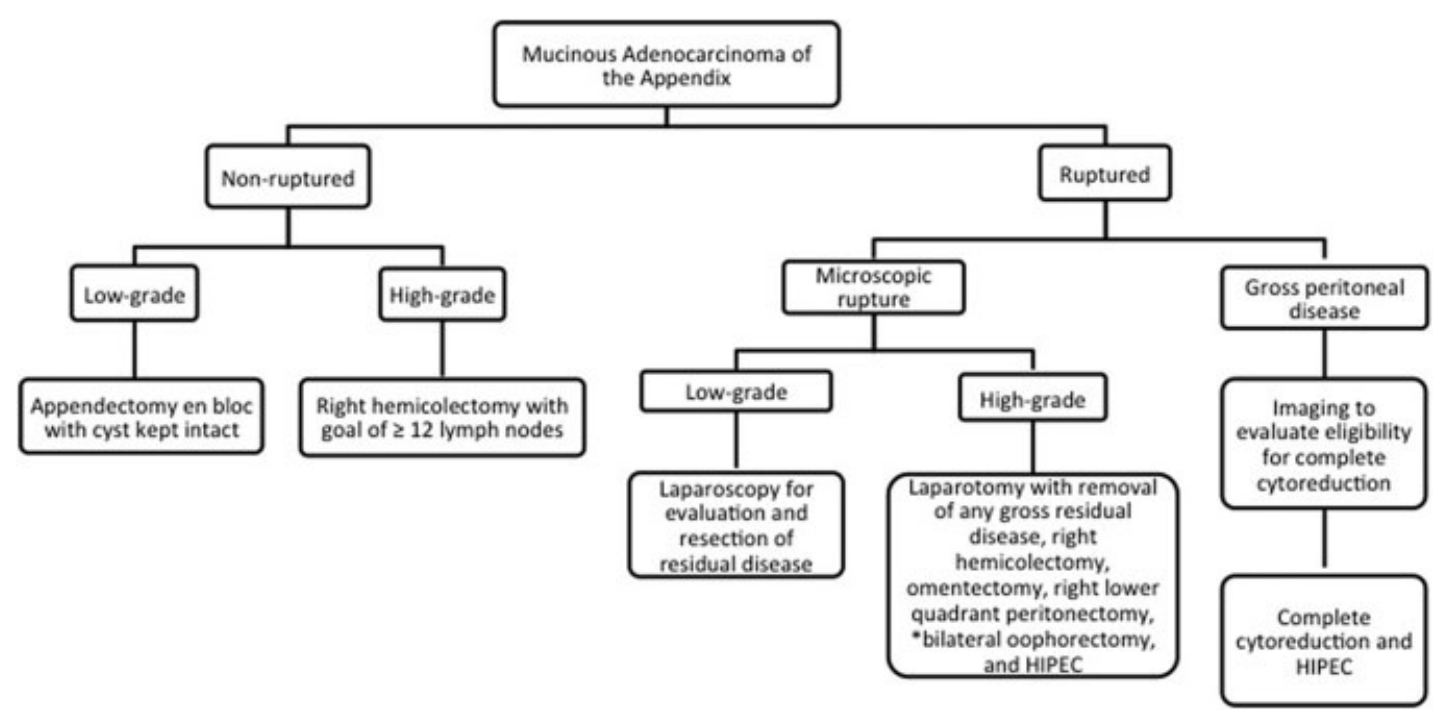

Fig. 3 Algorithm for management of mucinous appendiceal adenocarcinoma. HIPEC, hyperthermic intraperitoneal chemotherapy. 


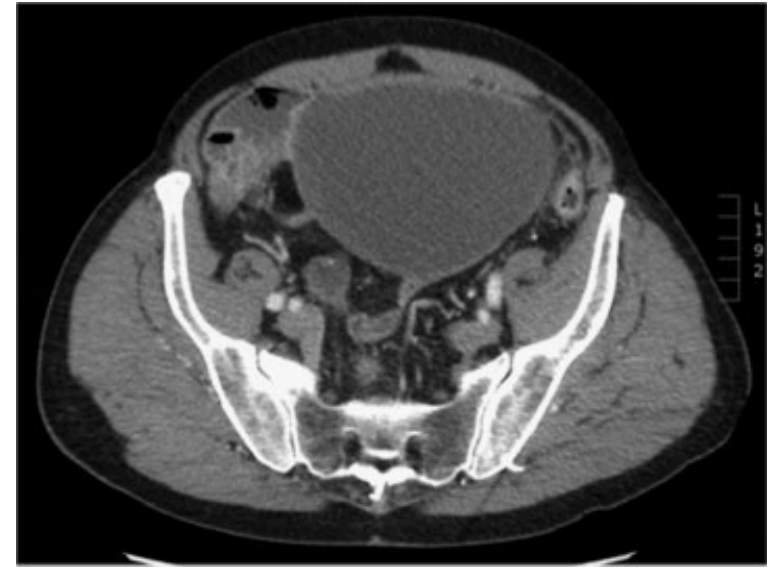

Fig. 4 CT scan demonstrating a large cystic lesion in a patient who presented with vague abdominal pain and increasing abdominal girth. This "cyst" was actually the patient's chronically dilated, mucin filled appendix which had not ruptured.

a patient like this with a large, mucin-filled, and chronically dilated but nonruptured appendix. Regardless of size, it is important to remove the appendix/cyst en bloc without spillage of mucin into the peritoneal cavity. For small lesions, this may be feasible to do laparoscopically, but for large lesions as illustrated in the figure, and open approach should be considered. For localized, nonruptured, low-grade MAA, appendectomy with en bloc resection of the cystic lesion is likely curative. If pathology demonstrates high-grade disease that is T2 or greater, right hemicolectomy is typically recommended due to the higher risk of lymph node involvement.

In cases where peritoneal dissemination has occurred, whether gross or microscopic, regional metastasis is present and regional therapy is indicated. The optimal therapy is complete surgical cytoreduction (defined as removal of all gross disease or reduction of tumor deposits to $\leq 2.5 \mathrm{~mm}$ in thickness) in combination with IP chemotherapy. Cytoreduction routinely involves complete omentectomy and right lower quadrant peritonectomy. Bilateral oophorectomy is also recommended, especially in postmenopausal women, as the ovaries are a frequent site of metastasis and recurrence if not removed. Women of childbearing age, in the absence of gross disease, may elect for preservation of the ovaries. If they do so, close follow-up with pelvic ultrasound in addition to standard cross-sectional imaging will allow for discrimination of benign and potentially malignant cystic lesions on the ovaries. Regardless of whether oophorectomy is performed, women of childbearing age undergoing cytoreduction and HIPEC should be preoperatively counseled regarding fertility preservation options, such as ovarian stimulation and egg retrieval.

IP chemotherapy is often administered in the form of intraoperative hyperthermic intraperitoneal chemotherapy (HIPEC), which is given as a single dose at the time of cytoreduction. Recently, the American Society of Peritoneal Surface Malignancy (ASPSM) agreed upon a standardized delivery method of IP chemotherapy for colorectal cancer with peritoneal dissemination, and this is the regimen used at most centers for appendiceal primary tumors as well. ${ }^{8}$ The consensus method is HIPEC delivered intraoperatively at the time of cytoreduction using a closed technique. The recommended agent is Mitomycin C(MMC) at a dose of $40 \mathrm{mg}$ in $3 \mathrm{~L}$ of perfusate. The perfusion is performed at an inflow temperature of $42^{\circ} \mathrm{C}$ and is continued for 90 minutes (30 $\mathrm{mg}$ of drug for first 60 minutes with additional $10 \mathrm{mg}$ added for last 30 minutes). Another option is early postoperative intraperitoneal chemotherapy (EPIC), which is administered in several doses during the first 1 to 7 days postoperatively, under normothermic conditions via an implanted subcutaneous port. Agents most commonly used are floxuridine, MMC, or 5-fluorouracil. ${ }^{9}$ No studies have shown one method to be superior to the other, but there is a randomized trial currently underway comparing the two techniques (NCT01815359).

For the patients whose diagnosis was made at appendectomy with pathology showing evidence of rupture or tumor cells outside of the appendix, cross-sectional imaging may not demonstrate any residual disease (PCI 0). In these cases, assessment of disease burden is best done by laparoscopy. If there is no gross tumor or mucin identified and pathology showed low-grade MAA, surveillance with examination and cross-sectional imaging is recommended. In this setting, IP chemotherapy is generally reserved for development of recurrent gross disease. There is a possibility that patients may not develop recurrent disease, so it is not necessary to expose patients to laparotomy, adhesion formation, and potential complications of IP chemotherapy in this setting.

For high-grade tumors with perforation and tumor cells outside of the appendix, laparoscopy or laparotomy with assessment for residual disease is warranted regardless of imaging findings. Complete cytoreduction of any gross residual tumor or mucin should be performed. This typically includes omentectomy and right lower quadrant peritonectomy, and may also include bowel resection, splenectomy, cholecystectomy, and salpingo-oophorectomy. Most specialists recommend administration of IP chemotherapy. At the time of cytoreduction, right hemicolectomy is used sparingly for low-grade MAA as lymph node metastasis is rare $6 \%$ of cases). ${ }^{10}$ In high-grade disease, nodal metastases are present in up to $20 \%$ of cases, so right hemicolectomy is a consideration; however, therapeutic benefit has not been demonstrated. ${ }^{10}$

The majority of patients with MAA present with more extensive, disseminated disease. In cases where crosssectional imaging demonstrates potentially resectable disease, laparoscopy is a useful adjunct to confirm eligibility for complete cytoreduction and to obtain tissue for histologic assessment if grade is not known. In patients with known low-grade disease, laparoscopy is not as important as there is a role for palliative debulking, even if complete cytoreduction cannot be achieved. Staged cytoreduction and HIPEC is also an option for patients with low-grade MAA where complete cytoreduction is technically possible, but cannot be completed safely in one operation due to prolonged operative time. In this setting, clearance of either the upper or lower abdomen is done at the time of the first operation. Patients are allowed to 
recover for 3 to 4 months, and then completion debulking of residual disease and IPC may be performed at a second operation.

For patients not eligible for complete cytoreduction, systemic chemotherapy with regimens utilized for metastatic colorectal cancer is the best initial therapy. Patients should undergo repeat imaging at intervals of 2 to 3 months to evaluate response to therapy. In some cases, patients will have a measurable response to therapy and become candidates for cytoreduction and IPC.

Even after complete cytoreduction and IPC, recurrence of MAA is common, in both low- and high-grade disease. In a recently published large series of patients, median diseasefree survival was 38.1 months for patients with low-grade MAA and 21.6 months for high-grade disease. ${ }^{10}$ Similarly, median overall survival at 5 years is on the order of 75 to $81 \%$ for low-grade MAA and 45 to $65 \%$ for high-grade disease. ${ }^{11-14}$ Low-grade MAA is not typically responsive to systemic therapy, so adjuvant treatment is uncommonly recommended. ${ }^{15}$ If recurrence develops, it is most often within the peritoneal cavity and is typically indolent and slowgrowing. Repeat debulking or complete cytoreduction/IPC are reasonable and have been reported result in long-term survival. Evidence suggests there is some improvement in progression-free survival with systemic chemotherapy for high-grade disease, although response rates are lower than hoped. ${ }^{15}$

\section{Goblet Cell Adenocarcinoma}

\section{Background and Epidemiology}

Goblet cell adenocarcinoma (GCA), also commonly referred to as "goblet cell carcinoid," is a rare tumor type accounting for approximately 14 to $19 \%$ of primary appendix cancers. ${ }^{1,16}$ GCA is a mixed tumor, containing both epithelial and neuroendocrine elements, and is characterized by the presence of intestinal-type goblet cells. Recent studies have shown that GCAs are actually closer to adenocarcinoma than neuroendocrine carcinoma in terms of both immunohistochemical profile and biologic behavior. ${ }^{10,17,18}$ The mean patient age at presentation of GCA is 52 years and there is no sex predilection. ${ }^{19}$ There are no established risk factors, although GCA has been associated with schistosomiasis in reports from China. ${ }^{20,21}$

\section{Presentation and Workup}

-Fig. 5 demonstrates a CT scan of a patient who presented with clinical findings of acute appendicitis. This is the most common presentation of GCA, occurring in up to $60 \%$ of cases. The tip of the appendix was dilated at $1.2 \mathrm{~cm}$ and was enhancing as shown in the figure. This finding was not specific for a mass, however, especially in the setting of periappendiceal fat stranding and inflammation. The patient underwent a laparoscopic appendectomy and was found to have a GCA.

Once the diagnosis of GCA is established, CT scan of the chest, abdomen, and pelvis is recommended for staging. Positron emission tomography (PET) has not been validated

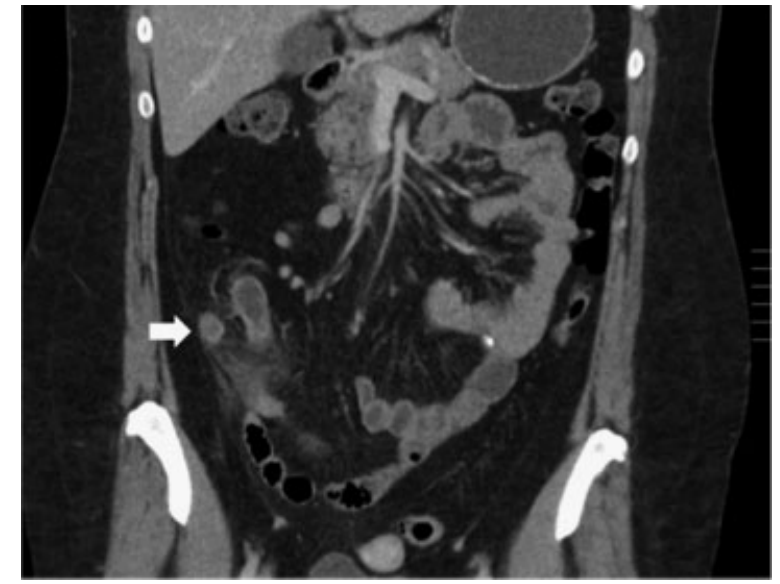

Fig. 5 CT scan demonstrating acute appendicitis. The tip of the appendix was somewhat enlarged and hyperenhancing. Final pathology revealed a $1.5-\mathrm{cm}$ goblet cell adenocarcinoma.

in this disease. The percentage of patients with stage IV disease at presentation varies considerably in different series but is thought to be greater than $50 \%$; the peritoneum is the most common site. If tumor markers are to be analyzed for potential utility in postoperative surveillance, CEA, CA 19-9, and CA-125, rather than chromogranin $A(\operatorname{CgA})$, are recommended. $^{19}$

\section{Management}

In cases of localized, primary appendiceal GCA, lymph node involvement is present in 20 to $40 \%$ of cases, so right hemicolectomy is recommended for adequate treatment and staging regardless of primary tumor size. ${ }^{16,17,19}$ As for colorectal adenocarcinoma, examination of at least 12 lymph nodes allows for optimal staging. If lymph node involvement is present, adjuvant systemic therapy with a 5-fluorouracilbased regimen is recommended for 6 months.

For GCA patients presenting with peritoneal-only metastatic disease, evidence suggests there is a role for cytoreduction and IPC. In a recent report of 45 patients with peritoneal-only metastasis of GCA undergoing evaluation for cytoreduction and HIPEC, complete cytoreduction was achieved in $71 \%$ of cases and HIPEC (with MMC or oxaliplatin) was administered in $80 \%{ }^{10}$ Lymph node metastases were present in 52\% of patients. For those patients who received complete cytoreduction and HIPEC, the estimated diseasefree and overall survival rates at 3 years were $43 \%$ and $63 \%$, respectively.

\section{Neuroendocrine Carcinoma}

\section{Background and Epidemiology}

Primary appendiceal neuroendocrine carcinoma (ANC), classically referred to as "appendiceal carcinoid," is considered a subtype of midgut neuroendocrine carcinoma, which can arise in the jejunum/ileum, the appendix, or the cecum. These tumors arise from enterochromaffin-like cells in the bowel wall and often produce serotonin. The published annual incidence of ANC is approximately 0.16 cases per 100,000 
people, but is likely underestimated as these tumors are often considered "benign" and are not included in cancer registries. The true incidence is unknown. Existing data suggest that ANCs exhibit a slight female sex predominance, and are more common in Caucasians and African Americans than in people of Asian/Pacific Island descent. ${ }^{22}$ The mean patient age at diagnosis is 38 to 48 years. ${ }^{1,4}$

\section{Presentation and Workup}

As for other appendiceal primary cancers, ANCs are most often diagnosed following appendectomy for appendicitis. In some cases, however, patients may report vague symptoms of nonspecific abdominal pain, bloating, and diarrhea. In exceedingly rare cases of advanced disease with distant metastasis, the most common site is the liver. These patients may present with carcinoid syndrome due to systemic effects of serotonin produced by the liver lesions. This is characterized by episodic flushing and diarrhea.
ANCs are classified by location within the appendix, size, and histologic features. The AJCC staging system for ANCs is shown in - Table $\mathbf{2 A}$, but it is of limited value because it is based only on size and does not account for tumor grade or other histologic features that are known to be important. Midgut neuroendocrine tumors are graded based on number of mitoses visualized on microscopy and on $\mathrm{K}_{\mathrm{i}}-67$ proliferation index. Low-grade tumors (G1) are defined as having fewer than two mitoses per 10 high-power fields (HPF) and $\mathrm{K}_{\mathrm{i}}$-67 index less than 3\%. Intermediate-grade tumors (G2) have 2 to 20 mitoses per $10 \mathrm{HPF}$ or $\mathrm{K}_{\mathrm{i}}-67$ index of 3 to $20 \%$. High-grade tumors (G3) have more than 20 mitoses per 10 HPF or $\mathrm{K}_{\mathrm{i}}-67$ index greater than $20 \%{ }^{23}$ The staging system adopted by the European Neuroendocrine Tumor Society (ENETS) (- Table 2B) accounts for tumor grade. ${ }^{19}$ In most cases, G1 and G2 tumors are well-differentiated, and G3 tumors are poorly differentiated and are the equivalent of "small- or large-cell" neuroendocrine carcinoma depending

Table 2 (A) AJCC and (B) ENETS staging systems for primary appendiceal neuroendocrine carcinoma

\begin{tabular}{|c|c|c|c|}
\hline \multicolumn{2}{|l|}{ A. AJCC } & \multicolumn{2}{|c|}{ B. ENETS } \\
\hline \multicolumn{4}{|l|}{ Tumor } \\
\hline- & - & $\mathrm{TX}$ & Primary tumor cannot be assessed \\
\hline- & - & T0 & No evidence of primary tumor \\
\hline T1a & $\leq 1 \mathrm{~cm}$ greatest diameter & $\mathrm{T} 1$ & $\begin{array}{l}\leq 1 \mathrm{~cm} \text { greatest diameter, invasion } \\
\text { to submucosa or muscularis propria }\end{array}$ \\
\hline $\mathrm{T} 1 \mathrm{~b}$ & $1-2 \mathrm{~cm}$ greatest diameter & - & \\
\hline $\mathrm{T} 2$ & $>2 \mathrm{~cm}$ but $\leq 4 \mathrm{~cm}$ or invasion of cecum & $\mathrm{T} 2$ & $\begin{array}{l}\leq 2 \mathrm{~cm} \text { invades submucosa, muscularis } \\
\text { propria, and/or } \leq 3 \mathrm{~mm} \text { of subserosa or } \\
\text { mesoappendix }\end{array}$ \\
\hline T3 & $>4 \mathrm{~cm}$ or extension to ileum & $\mathrm{T} 3$ & $\begin{array}{l}>2 \mathrm{~cm} \text { and/or }>3 \mathrm{~mm} \text { invasion of } \\
\text { subserosa or mesoappendix }\end{array}$ \\
\hline $\mathrm{T} 4$ & \multicolumn{3}{|l|}{ Perforates peritoneum or invades other organs } \\
\hline \multicolumn{4}{|l|}{ Nodes } \\
\hline- & - & NX & Nodes cannot be assessed \\
\hline No & \multicolumn{3}{|l|}{ No regional lymph node involvement } \\
\hline N1 & \multicolumn{3}{|l|}{ Metastasis to regional nodes } \\
\hline \multicolumn{4}{|c|}{ Metastasis } \\
\hline- & - & $\mathrm{MX}$ & Distant metastasis not assessed \\
\hline M0 & \multicolumn{3}{|l|}{ No distant metastasis } \\
\hline M1 & \multicolumn{3}{|l|}{ Distant metastasis } \\
\hline \multicolumn{4}{|l|}{ Stage } \\
\hline- & - & 0 & Tis, N0, M0 \\
\hline I & T1, N0, M0 & 1 & T1, N0, M0 \\
\hline II & T2/3, N0, M0 & IIA & T2, N0, M0 \\
\hline \multirow[t]{2}{*}{ III } & T4, N0, M0 & IIB & $\mathrm{T} 3, \mathrm{~N} 0, \mathrm{M} 0$ \\
\hline & Any $\mathrm{T}, \mathrm{N} 1, \mathrm{M} 0$ & IIIA & $\mathrm{T} 4, \mathrm{~N} 0, \mathrm{M} 0$ \\
\hline IV & Any $\mathrm{T}$, any $\mathrm{N}, \mathrm{M} 1$ & IIIB & Any $\mathrm{T}, \mathrm{N} 1, \mathrm{M} 0$ \\
\hline I & T1, N0, M0 & IV & Any $\mathrm{T}$, any $\mathrm{N}, \mathrm{M} 1$ \\
\hline
\end{tabular}

Abbreviations: AJCC, American Joint Commission on Cancer; ENETS, European Neuroendocrine Tumor Society. 
on the appearance and size of the cells. Most ANCs are welldifferentiated, G1 or G2 tumors, and 70 to $80 \%$ are located at the tip of the appendix. ${ }^{19}$

\section{Management}

In cases of subcentimeter, G1 ANCs diagnosed on retrospective analysis of an appendectomy specimen as occurs in most cases, appendectomy with negative margins is considered curative and no further staging or postoperative surveillance is necessary. ${ }^{19,23,24}$ Overall survival is essentially $100 \%$ in this group of patients.

Recommendations are less clear in patients with tumors sized greater than $1 \mathrm{~cm}$ but less than $2 \mathrm{~cm}$, and in those less than $1 \mathrm{~cm}$ with positive margins or other high-risk features. High-risk features include high mitotic rate or proliferative index (G2/3), positive surgical margin, location at the base of the appendix, invasion of the mesoappendix greater than $3 \mathrm{~mm}$, and lymphovascular invasion. If any of these features are present in tumors sized less than $2 \mathrm{~cm}$, patients should be counseled about a poorly characterized but possibly increased risk of recurrence. Laparoscopic right hemicolectomy should be considered for completion resection and appropriate staging, especially for young patients and those without comorbidities in whom the operation would be lower risk. Alternatively, cross-sectional imaging can be considered as means of assessing for locoregional and distant metastasis if patients do not wish to undergo a staging surgical procedure.

For ANCs greater than $2 \mathrm{~cm}$ in diameter, the risk of metastasis is on the order of 25 to $40 \%$, so right hemicolectomy is recommended. ${ }^{19,25,26}$ In the absence of lymph node involvement, even if the primary tumor was sized greater than $2 \mathrm{~cm}$, it is unclear whether specific follow-up or surveillance imaging is necessary. For cases with nodal or other resected locoregional or distant metastasis, however, long-term follow-up is advised.

For the exceedingly rare cases of ANC with distant metastases at presentation, consideration for surgical treatment is reasonable if the metastatic lesion or lesions are limited and are resectable. This is a slow-growing, indolent tumor type so metastasectomy may provide benefit in terms of disease free and possibly overall survival, although this has not been definitively shown. For extensive, unresectable liver metastases or patients who are not fit for surgery, somatostatin analogues have been shown to improve progression-free survival for midgut neuroendocrine carcinomas. ${ }^{27} \mathrm{CgA}$ can be monitored to assess for response along with interval imaging. Alternative systemic treatment options include peptide receptor radionuclide therapy and Everolimus. ${ }^{19,23}$

\section{Summary and Surveillance Recommendations}

In summary, primary cancers of the appendix are histologically diverse. Classification of these tumors has historically been confusing due to nonstandardized nomenclature, but they can be broadly classified as colonic-type or mucinous adenocarcinoma, GCA, or ANC. Appendectomy alone may be adequate therapy for early-stage tumors in all subtypes except GCA. For GCA and locally advanced adenocarcinoma or ANC, completion staging is considered, followed by right hemicolectomy, cytoreductive surgery and IPC, or definitive systemic chemotherapy as indicated.

After curative-intent treatment, patients with GCA, T2 or greater colonic-type adenocarcinoma, mucinous adenocarcinoma with peritoneal dissemination, and ANC with lymph node involvement or size greater than $2 \mathrm{~cm}$ should be followed with history and physical and cross-sectional imaging. Given the rarity of primary appendiceal cancer and the lack of established practice guidelines, patients with advanced disease should be treated at specialized centers with a multidisciplinary team approach.

\section{References}

1 McCusker ME, Coté TR, Clegg LX, Sobin LH. Primary malignant neoplasms of the appendix: a population-based study from the surveillance, epidemiology and end-results program, 1973-1998. Cancer 2002;94(12):3307-3312

2 Nitecki SS, Wolff BG, Schlinkert R, Sarr MG. The natural history of surgically treated primary adenocarcinoma of the appendix. Ann Surg 1994;219(1):51-57

3 Alakus H, Babicky ML, Ghosh P, et al. Genome-wide mutational landscape of mucinous carcinomatosis peritonei of appendiceal origin. Genome Med 2014;6(5):43

4 Benedix F, Reimer A, Gastinger I, Mroczkowski P, Lippert H, Kube R; Study Group Colon/Rectum Carcinoma Primary Tumor. Primary appendiceal carcinoma-epidemiology, surgery and survival: results of a German multi-center study. Eur J Surg Oncol 2010; 36(8):763-771

5 Davison JM, Choudry HA, Pingpank JF, et al. Clinicopathologic and molecular analysis of disseminated appendiceal mucinous neoplasms: identification of factors predicting survival and proposed criteria for a three-tiered assessment of tumor grade. Mod Pathol 2014;27(11):1521-1539

6 Jacquet P, Sugarbaker PH. Clinical research methodologies in diagnosis and staging of patients with peritoneal carcinomatosis. Cancer Treat Res 1996;82:359-374

7 Low RN, Barone RM. Combined diffusion-weighted and gadolinium-enhanced MRI can accurately predict the peritoneal cancer index preoperatively in patients being considered for cytoreductive surgical procedures. Ann Surg Oncol 2012;19(5):1394-1401

8 Turaga K, Levine E, Barone R, et al. Consensus guidelines from The American Society of Peritoneal Surface Malignancies on standardizing the delivery of hyperthermic intraperitoneal chemotherapy (HIPEC) in colorectal cancer patients in the United States. Ann Surg Oncol 2014;21(5):1501-1505

9 Kelly KJ, Nash GM. Peritoneal debulking/intraperitoneal chemotherapy-non-sarcoma. J Surg Oncol 2014;109(1):14-22

10 McConnell YJ, Mack LA, Gui X, et al. Cytoreductive surgery with hyperthermic intraperitoneal chemotherapy: an emerging treatment option for advanced goblet cell tumors of the appendix. Ann Surg Oncol 2014;21(6):1975-1982

11 Baratti D, Kusamura S, Nonaka D, et al. Pseudomyxoma peritonei: clinical pathological and biological prognostic factors in patients treated with cytoreductive surgery and hyperthermic intraperitoneal chemotherapy (HIPEC). Ann Surg Oncol 2008;15(2): 526-534

12 Chua TC, Moran BJ, Sugarbaker PH, et al. Early- and long-term outcome data of patients with pseudomyxoma peritonei from appendiceal origin treated by a strategy of cytoreductive surgery and hyperthermic intraperitoneal chemotherapy. J Clin Oncol 2012;30(20):2449-2456 
13 Chua TC, Yan TD, Smigielski ME, et al. Long-term survival in patients with pseudomyxoma peritonei treated with cytoreductive surgery and perioperative intraperitoneal chemotherapy: 10 years of experience from a single institution. Ann Surg Oncol 2009;16(7):1903-1911

14 El Halabi H, Gushchin V, Francis J, et al. The role of cytoreductive surgery and heated intraperitoneal chemotherapy (CRS/HIPEC) in patients with high-grade appendiceal carcinoma and extensive peritoneal carcinomatosis. Ann Surg Oncol 2012;19(1): 110-114

15 Blackham AU, Swett K, Eng C, et al. Perioperative systemic chemotherapy for appendiceal mucinous carcinoma peritonei treated with cytoreductive surgery and hyperthermic intraperitoneal chemotherapy. J Surg Oncol 2014;109(7):740-745

16 Turaga KK, Pappas SG, Gamblin T. Importance of histologic subtype in the staging of appendiceal tumors. Ann Surg Oncol 2012;19(5): 1379-1385

17 Tang LH, Shia J, Soslow RA, et al. Pathologic classification and clinical behavior of the spectrum of goblet cell carcinoid tumors of the appendix. Am J Surg Pathol 2008;32(10):1429-1443

18 van Eeden S, Offerhaus GJ, Hart AA, et al. Goblet cell carcinoid of the appendix: a specific type of carcinoma. Histopathology 2007; 51(6):763-773

19 Pape UF, Perren A, Niederle B, et al; Barcelona Consensus Conference participants. ENETS Consensus Guidelines for the management of patients with neuroendocrine neoplasms from the jejunoileum and the appendix including goblet cell carcinomas. Neuroendocrinology 2012;95(2):135-156
20 Jiang Y, Long H, Li T, Wang W, Liu H, Zhang X. Schistosomiasis may contribute to goblet cell carcinoid of the appendix. J Parasitol 2012;98(3):565-568

21 Jiang Y, Long H, Wang W, Liu H, Tang Y, Zhang X. Clinicopathological features and immunoexpression profiles of goblet cell carcinoid and typical carcinoid of the appendix. Pathol Oncol Res 2011; 17(1):127-132

22 Yao JC, Hassan M, Phan A, et al. One hundred years after "carcinoid": epidemiology of and prognostic factors for neuroendocrine tumors in 35,825 cases in the United States. J Clin Oncol 2008; 26(18):3063-3072

23 Boudreaux JP, Klimstra DS, Hassan MM, et al; North American Neuroendocrine Tumor Society (NANETS). The NANETS consensus guideline for the diagnosis and management of neuroendocrine tumors: well-differentiated neuroendocrine tumors of the jejunum, ileum, appendix, and cecum. Pancreas 2010;39(6):753-766

24 Murray SE, Lloyd RV, Sippel RS, Chen H, Oltmann SC. Postoperative surveillance of small appendiceal carcinoid tumors. Am J Surg 2014;207(3):342-345, discussion 345

25 Fornaro R, Frascio M, Sticchi C, et al. Appendectomy or right hemicolectomy in the treatment of appendiceal carcinoid tumors? Tumori 2007;93(6):587-590

26 Moertel CG, Weiland LH, Nagorney DM, Dockerty MB. Carcinoid tumor of the appendix: treatment and prognosis. N Engl J Med 1987;317(27):1699-1701

27 Arnold R, Rinke A, Klose KJ, et al. Octreotide versus octreotide plus interferon-alpha in endocrine gastroenteropancreatic tumors: a randomized trial. Clin Gastroenterol Hepatol 2005;3(8):761-771 